\title{
Reply to: lodine Nutrition and Thyroid Function in Pregnant Women Exposed to Different lodine Sources
}

\author{
Yanting Chen ${ }^{1} \cdot$ Wen Chen $^{1} \cdot$ Wanqi Zhang ${ }^{1}$ \\ Published online: 13 July 2019 \\ (C) Springer Science+Business Media, LLC, part of Springer Nature 2019
}

We are grateful to Dr. Zheng for the comments on our work: Iodine Nutrition and Thyroid Function in Pregnant Women Exposed to Different Iodine Sources (https://doi.org/10. 1007/s12011-019-01719-1) [1].

The first is that it is unclear if the authors excluded $\mathrm{Tg}$ values of women positive for thyroid antibodies in their $\mathrm{Tg}$ analysis. The percentages of women positive for thyroid antibodies were $3.7 \%$ and $3.3 \%$ in Tianjin and Shandong, respectively [2]. We presented the median Tg value of pregnant women in Tianjin and Shandong which were not positive for thyroid antibodies. CRM-457 was applied in the reagent kit for measurement of Tg via IMMULITE 2000. In this article, we aimed at evaluating the changes of median levels of thyroid function exposed to different iodine intakes but not only $\mathrm{Tg}$, the question mentioned by Dr. Zheng may be addressed in further study.

The second is there was a discrepancy in the categorisation of iodine status between urinary iodine concentration (UIC) and iodine-to-creatinine (UI/Cr) ratio. UIC was chosen to clarify the iodine status of pregnant women, which is recommended to evaluate iodine nutrition of pregnant women by $\mathrm{WHO} /$ UNICEF/ICCIDD [2]. The UI/Cr ratio $(121 \mu \mathrm{g} / \mathrm{g})$ was presented to show the median level of pregnant women in our study, and whether the value of UI/Cr ratio indicated iodine deficiency is still uncertain because no standard was established yet [3]. Therefore, no discrepancy existed in the categorisation of iodine status between UIC and UI/Cr ratio.
Although the iodine status of pregnant women was adequate, while part of them were still iodine deficiency, further study about indicators of iodine status is needed to carry out to assess the iodine nutrition properly and accurately.

\section{Compliance with Ethical Standards}

Conflict of Interest The authors declare that they have not conflict of interest.

\section{References}

1. Chen Y, Chen W, Du C, Fan L, Wang W, Gao M, Zhang X, Cui T, Hao Y, Pearce EN, Wang C, Zhang W (2018) Iodine nutrition and thyroid function in pregnant women exposed to different iodine sources. Biol Trace Elem Res. https://doi.org/10.1007/s12011-018$1530-8$

2. WHO/UNICEF/ICCIDD (2007) Assessment of iodine deficiency disorders and monitoring their elimination: a guide for programs managers, 3rd edn. World Health Organization, Geneva http:// www.ign.org/p142003094.html

3. Ma ZF, Venn BJ, Manning PJ, Cameron CM, Skeaff SA (2018) The sensitivity and specificity of thyroglobulin concentration using repeated measures of urinary iodine excretion. Eur J Nutr 57(4): $1313-1320$

Publisher's Note Springer Nature remains neutral with regard to jurisdictional claims in published maps and institutional affiliations.

Wanqi Zhang

wqzhang@tijmu.edu.cn

1 Department of Nutrition and Food Hygiene, School of Public Health, Tianjin Medical University, Tianjin, China 